\title{
A Case of Reverse Dialysis: Bladder Perforation During Robotic Hysterectomy Presenting as Acute Renal Failure
}

\author{
Muhammad Adnan Sohail ${ }^{\mathrm{a}, \mathrm{d}}$, Junaid Nasir ${ }^{\mathrm{b}}$, Umaira Ikram ${ }^{\mathrm{c}}$
}

\begin{abstract}
Robotic surgery is gaining more fame due to its advantages over laproscopic surgery especially in gynecological procedures. Intra operative complications are far less in the former. Bladder perforation is a rare intra operative complication of robotic hysterectomy that can present as fatal electrolyte disturbances, sepsis and might even give a picture of acute renal failure. We present a case of a bladder perforation status post hysterectomy that arrived in emergency with the symptoms of nausea, vomiting and ascities. Her blood chemistry was consistent with acute renal failure but with no radiological evidence of kidney disease. Cystoscopy and ureterography revealed a bladder perforation. The clinical and lab picture was thought to be due to the same injury that might have caused the urea and creatinine to be absorbed in the peritoneal cavity. The diagnosis was supported by the fact that her symptoms and abnormal lab values reverted to normal after the repair of the injury. The case which we have presented is yet another in league to help physician making diagnosis of bladder injury when they see a patient with a rapid hypercreatininemia and ascities of short duration especially in the setting of a recent intra abdominal surgery.
\end{abstract}

Keywords: Hyperkalemia; Renal failure; Creatinine

Manuscript accepted for publication November 1, 2011

\footnotetext{
a'St. Mary's Hospital, Waterbury, CT USA 06706, USA

${ }^{\mathrm{b}}$ Harlem Hospital Center, New York, USA

${ }^{\mathrm{c}}$ Ross University, Roso, Dominica

${ }^{\mathrm{d}}$ Corresponding author: Muhammad Adnan Sohail, Department of Internal Medicine, 56 Franklin Street, Waterbury, CT USA 06706, USA. Email: adnanke@gmail.com
}

doi:10.4021/wjnu6e

\section{Introduction}

Robotic surgery has developed as a recent surgical approach especially in gynecological operations due to its significant advantages like freedom of motion in seven degrees [1], 3dimensional views, improved dexterity, tremor reduction, and ergonomic advantages $[2,3]$ with fewer complications. Its intra operative complications in radical hysterectomies are less as compared to the total laproscopic radical hysterectomies. Of these complications bladder injury is even rarer [4]. Bladder injuries can result in profound electrolyte disturbances, paralytic ileus, septicaemia and death [5]. We report a case of a bladder injury post robotic hysterectomy which presented as an apparent acute renal failure.

\section{Case Report}

A 44 years old female, status post robotic hysterectomy presented with nausea and 4 episodes of vomiting, three days after her procedure. The vomitus contained food particles and no blood was ever observed. She denied having diarrhea or any other constitutional symptoms. Review of her systems yielded no significant findings. She also had a history of tubal ligations 10 years back. She was non diabetic, non hypertensive but was allergic to amoxicillin. She denied having history of kidney disease in the family. She was on Ibuprofen $800 \mathrm{mg}$ p. o t. i. d and acetaminophen/oxycodone $325 / 5 \mathrm{mg}$ every four hours as needed for pain. On presentation her temperature was $97 \mathrm{~F}$, pulse was $111 / \mathrm{min}$, respiratory rate was 20/min and blood pressure was 116/62 $\mathrm{mmHg}$. Her oxygen saturation was $100 \%$ on room air. On general physical examination she was awake, alert and in no acute distress. On examination of her respiratory system the lungs were clear to auscultation bilaterally, there were no wheezes or ronchi. On HEENT exam, no pallor or jaundice was appreciated. Pupils were equal, round and reactive to light and accommodating. Cardiovascular exam showed S1 and S2 with no additional sounds or thrill. Tachycardia was the only significant finding in her exam. The abdomen was soft, with no guarding or rigidity, though it was tender at the site of 
entry of surgical ports for hysterectomy. Bowel sounds were present.

At the time of admission her sodium was $134 \mathrm{meq} / \mathrm{l}$, potassium was $7 \mathrm{meq} / \mathrm{l}$, chloride $101 \mathrm{meq} / \mathrm{l}$, bicarbonates 22 $\mathrm{mmol} / \mathrm{L}$. Her BUN was $43 \mathrm{mg} / \mathrm{dl}$ and creatinine was $5 \mathrm{mg} / \mathrm{dl}$. Her glucose was $111 \mathrm{mg} / \mathrm{dl}$, WBC count 8000/UL, hemoglobin $11.4 \mathrm{~g} / \mathrm{dl}$, hematocrit 33.8. Her EKG was unremarkable except sinus tachycardia with ventricular rate of hundred per min. Liver function tests were all within normal limits. Lactic acid was $1 \mathrm{mmol} / \mathrm{l}$. Serum lipase was normal as well. Urine analysis was normal. When she was discharged after her procedure three days ago, her baseline creatinine was 0.8 $\mathrm{mg} / \mathrm{dl}$, but within a course of 3 days she developed hypercreatininemia. She had hyperkalemia with the value of $7 \mathrm{meq} / \mathrm{l}$. In the emergency the patient received insulin with dextrose and Kayexalate to lower down her potassium. Her potassium came down to 4.9 and 4.6 in successive measurements. These abnormal labs presented the picture of an acute renal failure. She received Zofran that settled her nausea and vomiting and was further investigated for the deranged renal function tests. CT scan of abdomen and pelvis was done which could not find any obstructive lesion in the urinary tract or any other finding contributing in acute renal failure. There was no hydronephrosis. CT showed free intraperitoneal air and large amount of fluid in the peritoneal cavity. The fluid measured 2 Hounsfield units in density. The fluid and air were seen in the left lower abdominal wall. The cause for the ascities was investigated. LFTs were all within normal limits except for low albumin which was $3.1 \mathrm{~g} / \mathrm{dl}$. Ultrasound of abdomen was unremarkable except for the presence of intraperitoneal fluid. Her chest X-ray showed normal heart shadow and no pulmonary infiltrate was seen. There was free air under the right hemidiaphragm which was suggested to be post hysterectomy. The patient also reported bloating sensation in her belly. She had a recent surgery done, so, after ruling out other probable causes for her abnormal presentation, intra operative injury was suspected. Hospital urologist was asked to see if there was any leak in her ureter or the urinary bladder. The patient had a cystocopy and ureterography done. A leak was found in the bladder wall which was about $3 \mathrm{~cm}$. The finding was reported and bladder injury was repaired with laprotomy incision at the same time. The fluid in the peritoneal cavity was taken out. She was started on antibiotics for 1 day and she coped well. Her BUN/Cr was checked three hours post surgical repair of urinary bladder and creatinine was 0.8 $\mathrm{mg} / \mathrm{dl}$. The patient was discharged to be followed up with the urologist and the gynecologist as an outpatient.

\section{Discussion}

The patient presentation in our case was not that alarming as to suspect any serious process going on in her in the beginning, which is why we suspected her vomiting to be due to post op pain or paralytic ileus. Though her complaints were relieved by the anti emetics, her labs came out alarming. She developed acute hyper creatininemia within 3 days and that might be the cause of her nausea and vomiting. The CT abdomen/pelvis could not find any obstructive lesions within the urinary tract. We also suspected acute renal failure due to drug toxicity as she was on Ibuprofen. But her features were not matching the typical presentation of interstitial nephritis. Her blood eosinophil count was normal and urine analysis was normal. There was no history of rash or fever [6]. Secondly in acute interstitial nephritis and as well as in glomerulonephritis of any type, the rate of rise of urea and creatinine is not that high. Hence glomerulonephritis was also suspended as a probable cause due to the same reason. Massive ascities in such acute setting was also an intriguing feature in this case. Her LFTs were normal, serum lipase was normal, no pleural effusion was found on CXR and her USG and CT both came up with the normal renal reports. The fact that she recently underwent hysterectomy and had massive ascities raised the suspicion of urinary tract injury. More over with this presentation, a rapid rise in serum creatinine was a diagnostic indicator of urinary tract injury itself [7] and also the fact that her abnormal labs reverted to normal after her bladder repair. Cystoscopy and ureterography was done to get the final diagnosis of perforated bladder. The most probable pathophysiology is the peritoneal auto dialysis of urea and creatinine. Urea and creatinine can get absorbed from the peritoneal cavity and raise the blood urea and creatinine levels [8]. This was experimentally proven by injecting a 100 $\mathrm{ml}$ urine volume in the peritoneal cavity of a dog and observing the rise in serum creatinine [8]. This increased absorption from the peritoneal cavity raised the blood levels of urea, creatinine and potassium and came up with the abnormal presentation.

By reporting this case we want to emphasize that the acute bladder injury due to a surgical procedure can show rapid changes in blood chemistry. The surgeons should keep an eye on post op picture of the serum electrolytes as that might give clue towards a serious issue with no serious presentation.

\section{Conflict of Interest}

We have nothing to disclose and have no conflict of interest.

\section{References}

1. Usui S, Inoue H, Yoshida T, Kudo SE, Iwai T. Preliminary report of multi degrees of freedom forceps for endoscopic surgery. Surg Laparosc Endosc Percutan Tech. 2004;14(2):66-72.

2. Stylopoulos N, Rattner D. Robotics and ergonomics. 
Surg Clin North Am. 2003;83(6):1321-1337.

3. Sroga J, Patel SD, Falcone T. Robotics in reproductive medicine. Front Biosci. 2008;13:1308-1317.

4. Kruijdenberg CB, van den Einden LC, Hendriks JC, Zusterzeel PL, Bekkers RL. Robot-assisted versus total laparoscopic radical hysterectomy in early cervical cancer, a review. Gynecol Oncol. 2011;120(3):334-339.

5. Thompson IM, Johnson EL, Ross G, Jr. The Acute Abdomen of Unrecognized Bladder Rupture. Arch Surg. 1965;90:371-374.
6. Neilson EG. Tubulointerstitial diseases. In: Goldman L, Ausiello D, eds. Cecil Medicine. 23rd ed. Philadelphia, Pa: Saunders Elsevier. 2007: chap 123.

7. Ratliff DA, Scoble JE. Spontaneous perforation of the bladder presenting as apparent acute renal failure. Br J Urol. 1987;60(4):369.

8. Shah PM, Kim KH, Ramirez-Schon G, Reynolds BM. Elevated blood urea nitrogen: an aid to the diagnosis of intraperitoneal rupture of the bladder. J Urol. 1979;122(6):741-743. 